\title{
EFECTOS DE LA COMUNICACIÓN EN UNA RED AD-HOC
}

Recibido: 3 de junio 2015- aceptado: 1 de diciembre 2015

Mayra Zurbaran ${ }^{1}$

Universidad del Norte

Paul Sanmartin ${ }^{1}$

Universidad del Norte

Keywords:

1. NS3, Ad Hoc, Node, Link, Simulator.

\section{Abstract}

The Ad-hoc networks is a revolution that benefits the community because of its decentralized architecture that facilitates many devices to communicate with each other, regardless whether they are mobile. This article presents the results of the effects of communication is presented in an ad hoc network, the simulation was divided into two stages, the first stage consists of two nodes which are separated deliberately causing the loss of signal and the second scenario provides that a node fails to study their behavior.

\section{Resumen}

NS3, Ad Hoc, Las redes Ad-hoc es una revolución que trae beneficios a la comunidad debido a su arquitectura descentralizada y que facilita que muchos dispositivos puedan comunicarse entre sí, sin importar que sean móviles. En este artículo se presenta los resultados de los efectos de comunicación en una red ad hoc, la simulación fue dividida en dos escenarios, en el primer escenario se compone de dos nodos los cuales se separan provocando adrede la pérdida de la señal y en el segundo escenario se establece que un nodo falle para estudiar su comportamiento. 


\section{INTRODUCCIÓN}

Una red móvil Ad-hoc (MANET) es una red inalámbrica en la que los servidores móviles son libres de formar conexiones dinámicas con otros que se encuentren dentro del radio de alcance sin ninguna infraestructura fija. Las redes Ad-hoc pueden funcionar sin conexión a internet, aunque también pueden hacerlo con conexión.

Debido a las conexiones dinámicas, la capacidad de los enlaces y la energía finita de cada nodo, es complicado garantizar una calidad de servicio (QoS) en una red MANET. Dada la naturaleza en cómo se conforman este tipo de redes, los nodos sirven como terminales y como switches.

Ya que los nodos no son estáticos, las conexiones entre ellos pueden variar. Si un grupo de nodos está conectado en un instante de tiempo $t_{1}$, en otro instante $t_{2}$ esto pudo cambiar y con esto varía la topología de la red. La mayoría de la veces los nodos establecen dinámicamente una ruta entre ellos y esto incrementa la dificultad en el trabajo sobre este tipo de redes.

La calidad del servicio es un aspecto de gran importancia en un red pues es un tipo de garantía que asegura un cierto grado de servicio que el sistema debe proveer dependiendo de cierto parámetros como: retraso, desempeño, fluctuación, pérdida de paquetes, latencia, tasa de perdida, tráfico en la red, ancho de banda disponible, etc. según [1]. En las redes MANET por los factores antes mencionados esto se dificulta hasta el punto en que dada una alta movilidad de los terminales es imposible ofrecer algún tipo de garantía [2]. En [1] se propone un algoritmo para mejorar la calidad del servicio en redes MANET.

El presente artículo consta de cinco secciones, en la sección II se hable sobre el ancho de banda en las redes ad-hoc, en la tercera sección la metodología, en la cuarta sección los resultados y luego las conclusiones.

\section{ANCHO DE BANDA EN REDES AD HOC}

Uno de los retos para estimar la calidad del servicio es la estimación del ancho de banda disponible (ABW) en la red. Al conocer el $A B W$ es posible realizar predicciones sobre QoS.

El tráfico en internet se ha incrementado gracias a la popularización de dispositivos como smartphones, tablets y laptops. Esta diversidad ha hecho más compleja la estructura de internet y haciendo del ABW una herramienta vital para los servicios en una red.

El ancho de banda disponible está determinado por el cuello de botella de la red, que es el que tiene el menor ancho de banda disponible. Herramientas como pathneck [3] puede decir donde se encuentra ese cuello de botella en el tramo. En [4] se propone un método para medir el ancho de banda disponible en múltiples partes de un tramo.

\section{Metodología}

Se realizaron dos laboratorios diferentes sobre redes adhoc usando la herramienta de simulación para redes NS3 (Network Simulator 3). El simulador de red NS3; versión siguiente al NS2, fue diseñado para ser escalable y modular.

Los nodos en el simulador son una representación de computadores reales, incluye el soporte para interfaces como sockets y dispositivos de red, el uso de direcciones IP, interfaces, entre otras.

\section{A. Escenario 1}

En este escenario (ver Figura 3.1) se muestran dos nodos conectados de modo ad-hoc, el nodo 1 empieza a moverse a una velocidad constante de $2 \mathrm{~m} / \mathrm{s}$ después de 30 segundos.

Para este escenario se necesitará de una velocidad de transmisión de $36 \mathrm{Mbps}$, se utilizan paquetes UDP con tamaño de 1400 bytes y un intervalo entre paquetes de 0,0002 segundos.

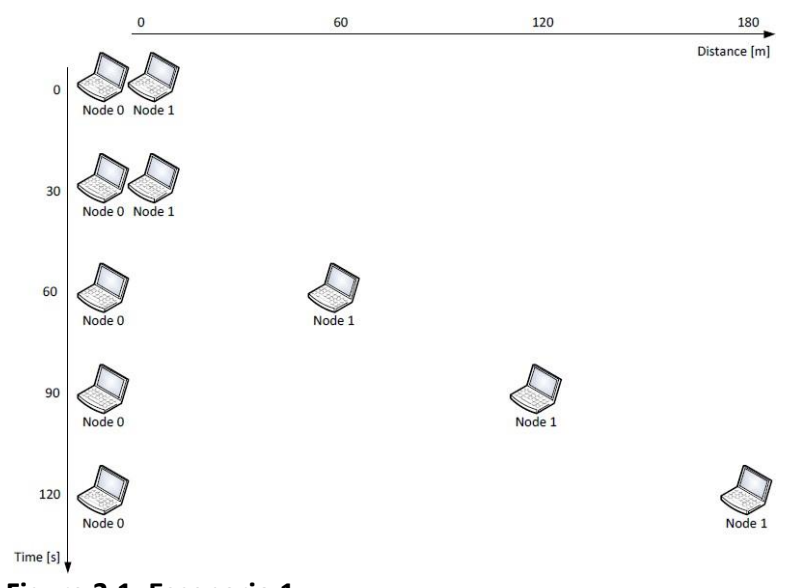

Figura 3.1: Escenario 1 
Tabla 3.1: Características del canal Escenario 1

\begin{tabular}{|l|l|}
\hline \multicolumn{1}{|c|}{ Atributo } & \multicolumn{1}{c|}{ Valor } \\
\hline Velocidad de Transmisión & $36 \mathrm{Mbps}$ \\
\hline Intervalo entre paquetes & $0.0002 \mathrm{seg}$ \\
\hline Numero de nodos & 2 \\
\hline Numero Máximo de paquetes & 1000000000 \\
\hline Tipo de paquetes & UDP \\
\hline Tamaño del paquete & 1400 bytes \\
\hline
\end{tabular}

\section{B. Escenario 2}

En este escenario la configuración de la red varía en cuanto a número de nodos con respecto al escenario 1 (ver Figura 3.2)

La comunicación entre el Nodo 0 y el nodo 3 Se realiza a través del nodo 1 . Después de 50 segundos, el nodo 1 falla y el algoritmo de enrutamiento envía los datos a través del nodo 2 .

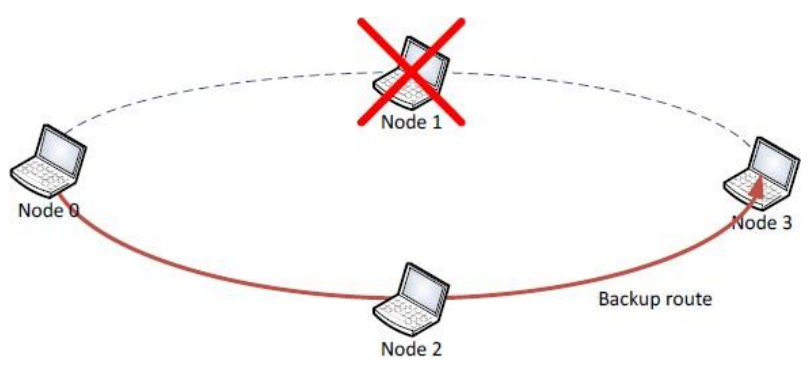

Figura 3.2: Escenario 2

Tabla 3.2 Características del canal

\begin{tabular}{|l|l|}
\hline \multicolumn{1}{|c|}{ Atributo } & \multicolumn{1}{c|}{ Valor } \\
\hline Velocidad de Transmisión & 10 y $30 \mathrm{Mbps}$ \\
\hline Intervalo entre paquetes & 0.0002 \\
\hline Numero de nodos & 4 \\
\hline $\begin{array}{l}\text { Número Máximo de } \\
\text { paquetes }\end{array}$ & 1000000000 \\
\hline Tipo de paquetes & UDP \\
\hline Tamaño del paquete & 1400 bytes \\
\hline Nodo emisor & Nodo 0 \\
\hline Nodo Receptor & Nodo 3 \\
\hline
\end{tabular}

\section{Resultados del escenario 1.}

Teniendo en cuenta que el nodo 1 es el emisor (ver Tabla 3.1) del escenario 1, en los resultados de la simulación podemos observar que este nodo empieza a enviar paquetes constantemente a partir del segundo 30 hasta el segundo 100 como se muestra en la Figura 4.1 .

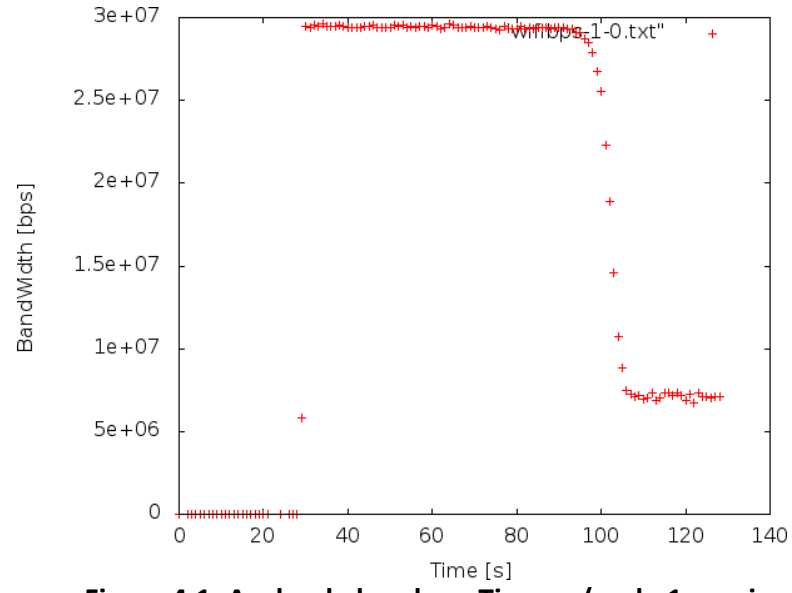

Figura 4.1: Ancho de banda vs Tiempo (nodo 1 - emisor).

De igual forma el nodo dos empieza a recibir paquetes a partir del segundo 30 tal y como se muestra en la Figura 4.2. Si observamos la Figura 4.1 notamos que el nodo 1 baja considerablemente su flujo de transmisión pero no para de transmitir hasta que termina la simulación. En cambio el nodo 0 desde ese mismo instante deja de recibir paquetes (ver Figura 4.2)

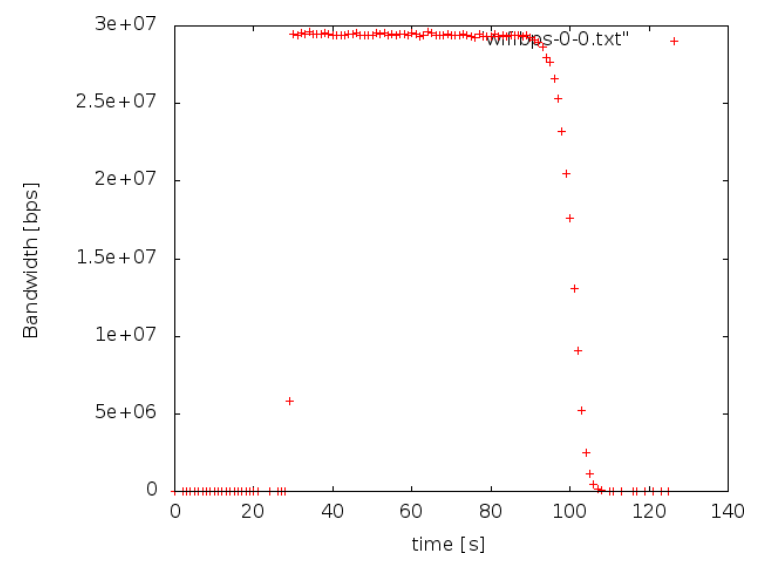

Figura 4.2: Ancho de banda vs Tiempo en el (nodo 0 - receptor) 


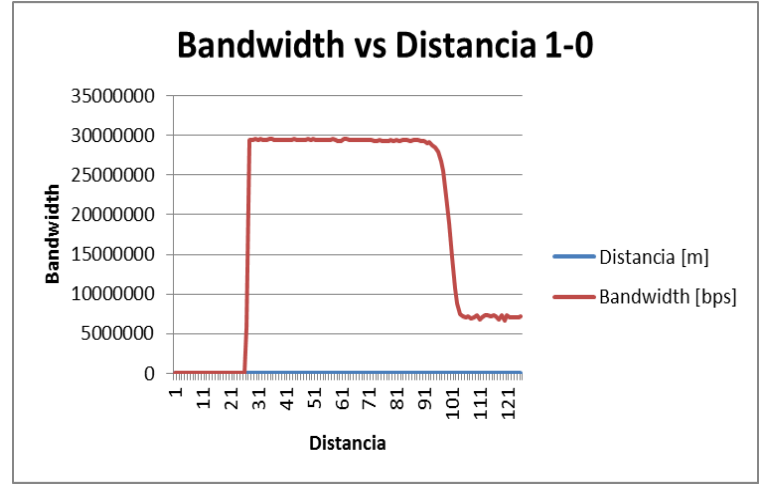

Figura 4.3: Ancho de banda vs Distancia (nodo 1 - emisor)

En la Figura 4.3 y 4.4 se evidencia que a partir de los 100 mts cuando el nodo 1 (emisor) tiende alejarse queda transmitiendo a una tasa por encima de los $5 \mathrm{Mbps}$ y el nodo 0 no prácticamente queda recibiendo cero paquetes (ver Figura 4.3)

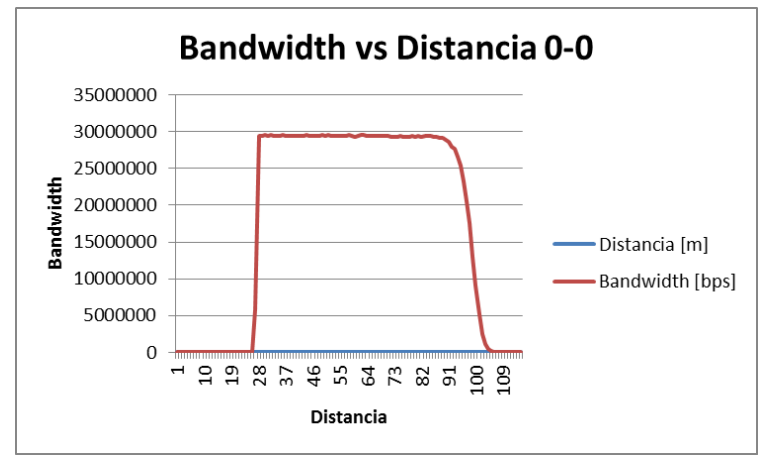

Figura 4.4: Ancho de banda vs Distancia (nodo 0 - receptor)

Las Figuras 4.5 y 4.6 evidencian que fueron enviados entre 2000 y 2500 paquetes desde los 30 hasta aproximadamente los 100 segundos

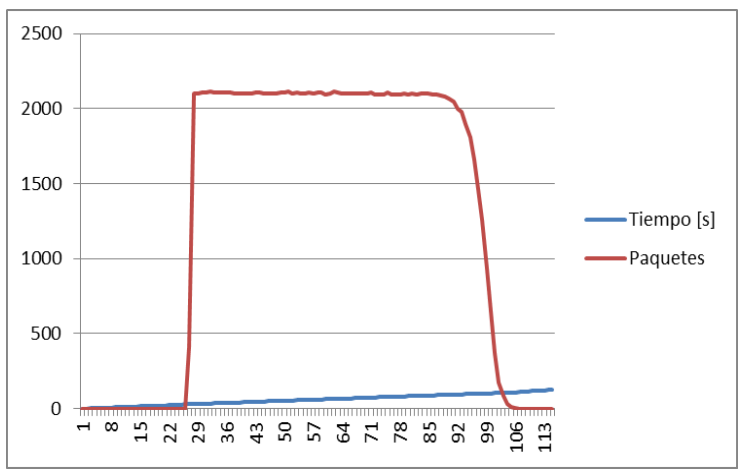

Figura 4.5: Número de paquetes vs Tiempo (nodo 0 - receptor).

Si miramos detenidamente el nodo 1 (quien es el emisor) en la Figuera 4.6, se nota que se queda transmitiendo por ensima de 500 paquetes.

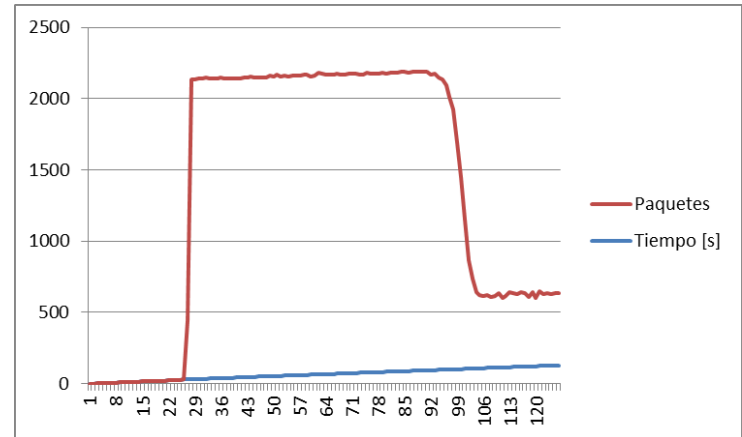

Figura 4.6: Ancho de banda vs Tiempo (nodo 1 -emisor)

\section{Resultados del escenario 2}

En el escenario 2 se realizaron dos pruebas: En un primer caso se aplica una velocidad de transmisión de 10 Mbps y en un segundo caso con velocidad de transmisión a $30 \mathrm{Mbps}$, teniendo la misma configuración de red para ambos casos tal y como se describió en la sección 3 (ver Tabla 3.2)

\section{Resultados con 10 Mbps: caso 1}

Al igual que en el escenario 1 , los primeros 30 segundos de la simulación se estipula para la convergencia en el enrutamiento. Los resultados en esta prueba muestran según la Figura 4.7 que se duplica el envío de los paquetes hasta el segundo 50. Sin embargo, el nodo receptor recibe un flujo de paquetes constante desde que empieza la transmisión, salvo cuando ocurre la caída del nodo 1 como muestra la Figura 4.8.

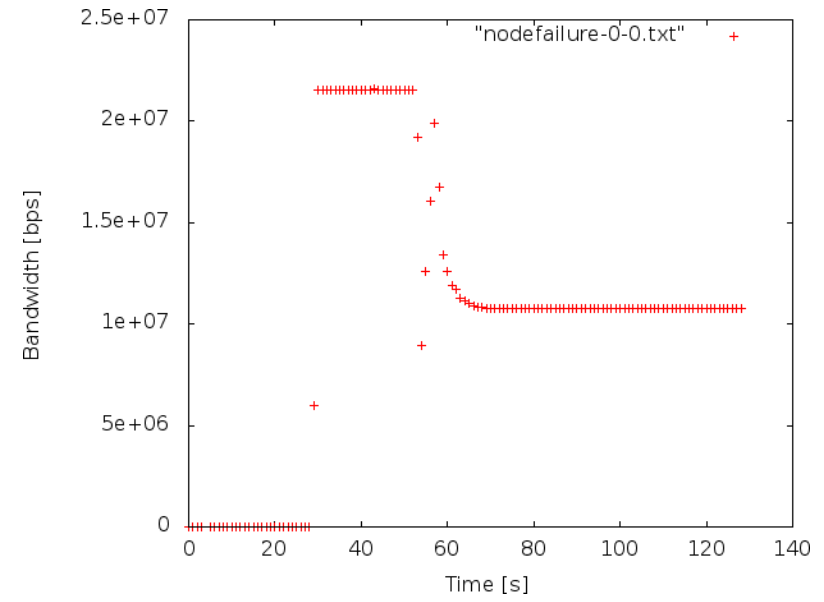

Figura 4.7: Ancho de Banda vs Tiempo en el nodo 0 (emisor)

A pesar de que el nodo 1 prácticamente desaparece de la red, el nodo receptor sigue recibiendo paquetes (ver 
Figura 4.7) debido que se utiliza otro camino con el nodo 2 de intermedio.

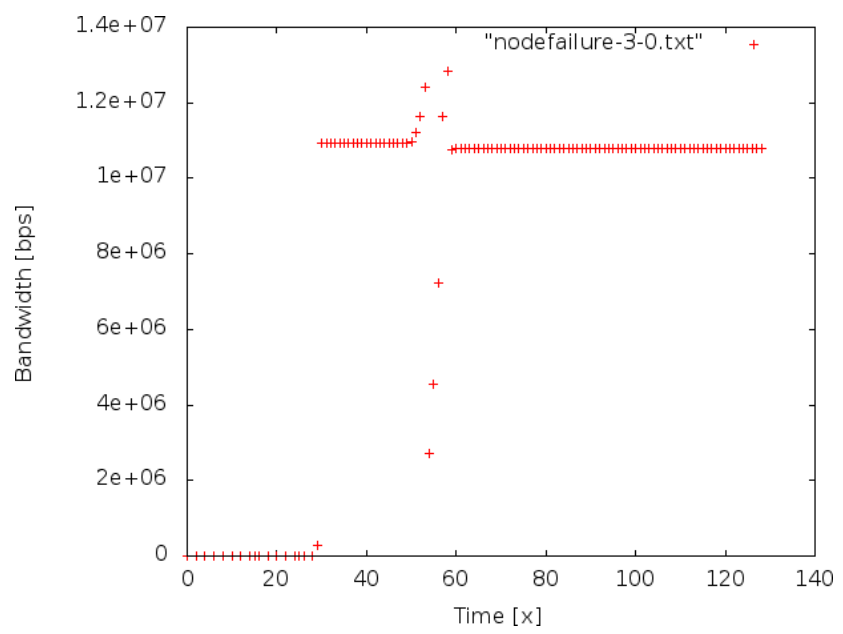

Figura 4.7: Ancho de Banda vs Tiempo (nodo 3 - receptor)

\section{Resultados con 30Mbps: caso 2}

La prueba realizada con una velocidad de transmisión de $30 \mathrm{Mbps}$ arroja unos resultados muy particulares. En contraste con los resultados del caso 1 , aquí nuca se alcanza el máximo ancho de banda permitido, por el contrario el nodo 0 (emisor) deja de transmitir de forma regular un poco después del segundo 60 (salvo cuando se cae el nodo 1) hasta que termina la simulación, tal y como se muestra en la Figura 4.8 y 4.9.

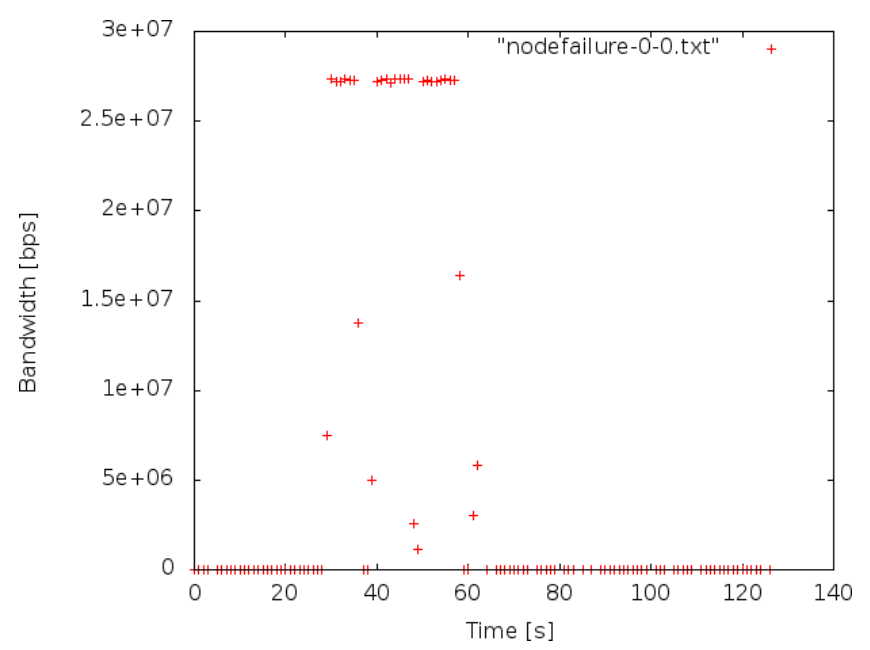

Figura 4.8. Gráfica de Ancho de Banda vs Tiempo (nodo 0 emisor).

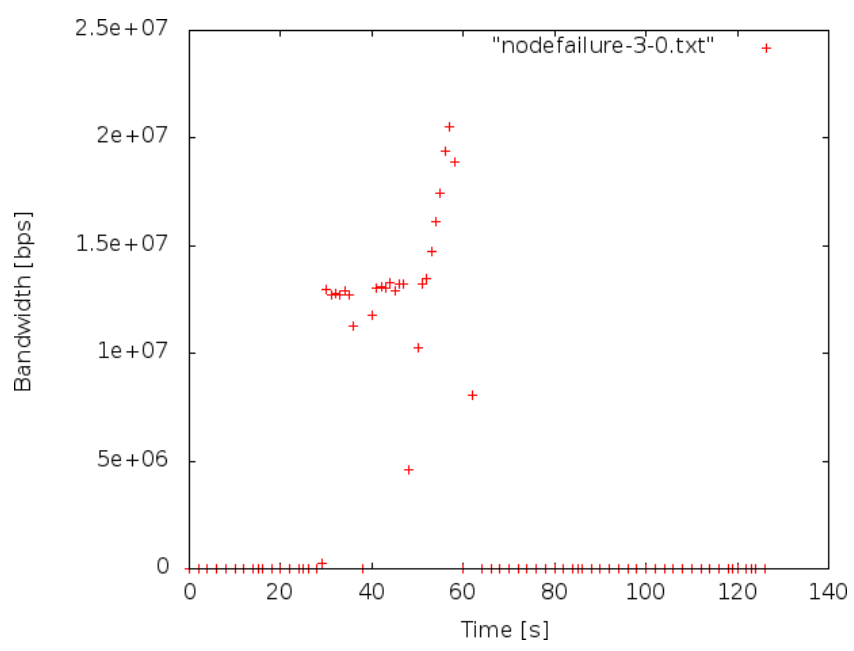

Figura 4.9: Ancho de Banda vs Tiempo (nodo 3 - receptor)

\section{CONCLUSIONES}

A través de la herramienta NS3, se simularon dos escenarios basados en redes ad hoc: En el primer escenario se pudo notar que a medida que se iban separando los nodos, al mismo tiempo se iba perdiendo alcance de la señal, motivo por el cual desde el segundo 100 el nodo 0 dejó de recibir paquetes, sin embargo, mientras los nodos tenían alcance, desde el segundo 30 la transmisión de los paquetes fue efectiva a una velocidad de transmisión de 36 Mbps.

En el segundo escenario se realizaron las pruebas con dos velocidades de transmisión distintas: 10 y $30 \mathrm{Mbps}$. En el caso 1 solo se pierden paquetes en el momento en que el nodo 1 sale de red como muestra la Figura 4.7. En contraste, en el caso 2 hubo más pérdida de paquetes, según lo mostrado en la Figura 4.9 solo hubo recepción de paquetes en el rango de los 30 y los 60 segundos.

Cabe destacar que en el escenario 2 para ambos casos, una vez el nodo uno desapareció de la red, los paquetes fueron transmitidos por otro camino que este caso usando como intermedio el nodo 2, evidenciando el trabajo del protocolo de enrutamiento. 
[1] Z. Rahaman and A. Das, "An algorithm to enhance the quality of service in mobile adhoc network," in Parallel Distributed and Grid Computing (PDGC), 2012 2nd IEEE International Conference on, 2012, pp. 355-358.

[2] Kazantzidis M., Gerla M., and Lee S., "Permissible throughput network for adaptive multimedia in AODV MANETs" Proceeding of the IEEE ICC 200 I pages 1352-1356

[3] N. Hu, L. E. Li, Z. M. Mao, P. Steenkiste, and J. Wang, "Locating Internet Bottlenecks: Algorithms, Measurements, and Implications," in Proceedings of ACM SIGCOMM 2004, pp. 41-54, September 2004

[4] Koitani, K.; Hasegawa, G.; Murata, M., "Measuring available bandwidth of multiple parts on end-to-end network path," Communications Quality and Reliability (CQR), 2012 IEEE International Workshop Technical Committee on , vol., no., pp.1,6, 15-17 May 2012.

[5] G. Carneiro. (2010). NS-3: Network Simulator 3. In UTM Lab Meeting April (Vol. 20). 